Hakam H. Alfakhry

BDS, MSc. (Asst. Prof.)

Saba H Al Zubaidi

BDS, MSc. (Asst. Prof.)

Anas AL mukhtar

BDS, MSc. PhD. (Lec.)

\section{Treatment of Horizontally Impacted Lower Cuspid with Two Roots Anatomical anomaly (Case Report)}

Depart. of Pedodontic, Ortho. and Preventive Dentistry

College of Dentistry, University of Mosul

Depart. of Pedodontic, Ortho. and Preventive Dentistry

College of Dentistry, University of Mosul

Depart. of Pedodontic, Ortho. and Preventive Dentistry

College of Dentistry, University of Mosul

\begin{abstract}
الخلاصة

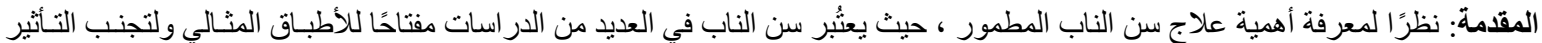

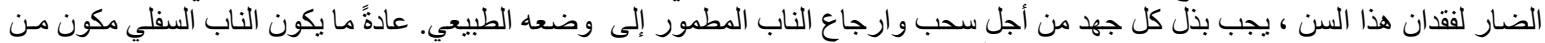

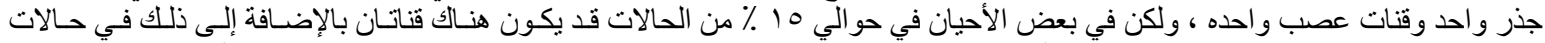

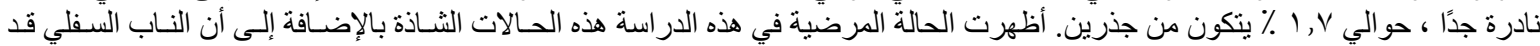

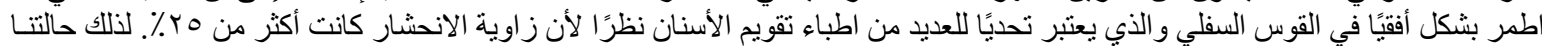

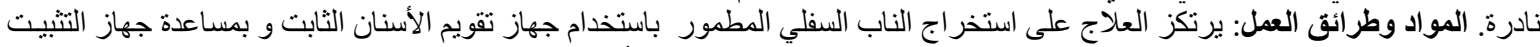

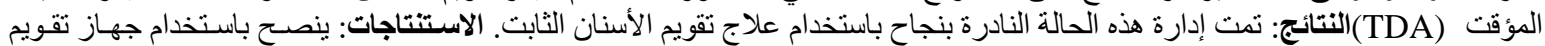
الاسنان الثابت في علاج حالات عدم بزوغ سن الناب و المطمور في الفكا السفلي و المتأثر أفقياً.
\end{abstract}

\begin{abstract}
Background: As the knowledge of the important of treatment the impacted canine since in many studies was considered as the key for ideal occlusion and to avoid the deleterious effect of lost, every effort must be done in order to retract and bring the impacted canine in to it is normal position. lower cuspid usually of a single rooted, but some time in about $15 \%$ of cases may have two canals in addition to that in a very rare cases approximately $1.7 \%$ associated with two roots. Our case showed these anomalies in addition to that the canine was horizontally impacted in the lower arch which is consider as challenge for many orthodontic since the angular of impaction was more than 25\%. therefore, our case is a rare. Materials and Method: The treatment focus on the orthodontically extrusion and up righting of the impacted canine by using fix orthodontic appliance with the aid of traditional anchorage device (TDA). Result: this rare case was successfully managed with fix orthodontic treatment. Conclusion: Orthodontic extrusion of horizontally impacted incomplete transposition mandibular canine should be considered for similar cases.
\end{abstract}

Keywords: Impacted, Lower Cuspid, Roots anomaly.

Alfakhry HH., Al Zubaidi SH., AL Mukhtar A. Treatment of Horizontally Impacted Lower Cuspid with Two Roots Anatomical anomaly (Case Report). AL-Rafidain Dent J. 2021; 21(2):215-227.

DOI: 10.33899/rdenj.2021.169268 C2020, College of Dentistry, University of Mosul.

Received: 2/10/2020 Sent to Referees: 5/10/2020

This is an open access article under the CC BY 4.0 license
Accepted for Publication: 5/11/2020 (http://creativecommons.org/licenses/by/4.0/). 


\section{INTRODUCTION}

Delay in normal eruption time of teeth or obstruction to normal eruption path in a way that tooth eruption is partially or totally prevented is described as impacted tooth ${ }^{(1)}$. Any tooth in the dental Arch may be impacted, however, Mandibular third molars, maxillary third molars and maxillary canines have the highest incidence, three times higher rate in female patients ${ }^{(2,3)}$. Mandibular canine impaction less frequent ${ }^{(4,5)}$. A low prevalence of impacted lower canine has been reported by many studies with the percentage ranging between $0.07 \%$ and $0.92 \%$ of a total number of teeth impaction ${ }^{(6-8)}$.The location of impacted lower canines are more likely to be on the labial aspect of the dental arch when compared to maxillary canines ${ }^{(9,10)}$.

Inadequate space, supernumerary teeth, premature loss or prolong retention of deciduous dentition, hereditary factors, tumours, cysts and trauma are the most reported causes of mandibular canine impaction $^{(11,12)}$.

The effects of impacted teeth include ecchymosis of the soft tissues, infection, paresthesia and damage to adjacent structures ${ }^{(13,14)}$ further, the presence of an impacted canine may cause resorption of the adjacent tooth, most likely a lateral incisor or the canine itself may undergo cystic changes ${ }^{(15,16)}$.

several options proposed for treatment of impacted teeth to avoid it is deleterious effect includes the following no treatment, autotransplantation of the canine, extraction of the impacted canine and movement of a first premolar in its position, extraction of the canine and posterior segmental osteotomy to move the buccal segment mesially to close the residual space, prosthetic replacement of the canine, Surgical exposure of the canine and orthodontic treatment to bring the tooth into the line of occlusion. This is obviously the most alternative treatments for impacted teeth ${ }^{(17)}$.

Normally, the mandibular canine is a single-rooted tooth with one root canal (18). Variations in the internal and external morphology of the mandibular canine have also been documented in the literature. Approximately $15 \%$ of the cases reported the presence of two canals in the lower canine. On very rare occasions, the presence of two different angulated roots was reported ${ }^{(19)}$. The anatomical variation of two roots with two separate root canals in lower canine is rare and literature search has revealed $5 \%, 1 \%$ and $1.2 \%$ cases with two roots and two root canals respectively (20).

\section{MATERIALS AND METHODS}

\section{Pretreatment Assessment:}

The study was approved by Research Ethics Committee board (University of Mosul, College of Dentistry, REC reference No. POP/H.5/11/20).

A 17-year-old female presented to our care at the clinic in the city of Zakho, Iraq. The chief complaint was crowded upper anterior teeth with an anterior open bite. The patient presented with good gen- 
eral health with no history of related systemic illness or injury. Extra-oral examination (Figure 1) showed a straight facial profile, competent lips, with no significant facial asymmetry. Intra-oral examination (Figure 1,2) revealed that all teeth were present except the left lower canine, left lower second molar and all third molar. Class I molar and canine relationship were recorded on the right segment whereas class II molar relation was found on the left segment with anterior open bite, over-
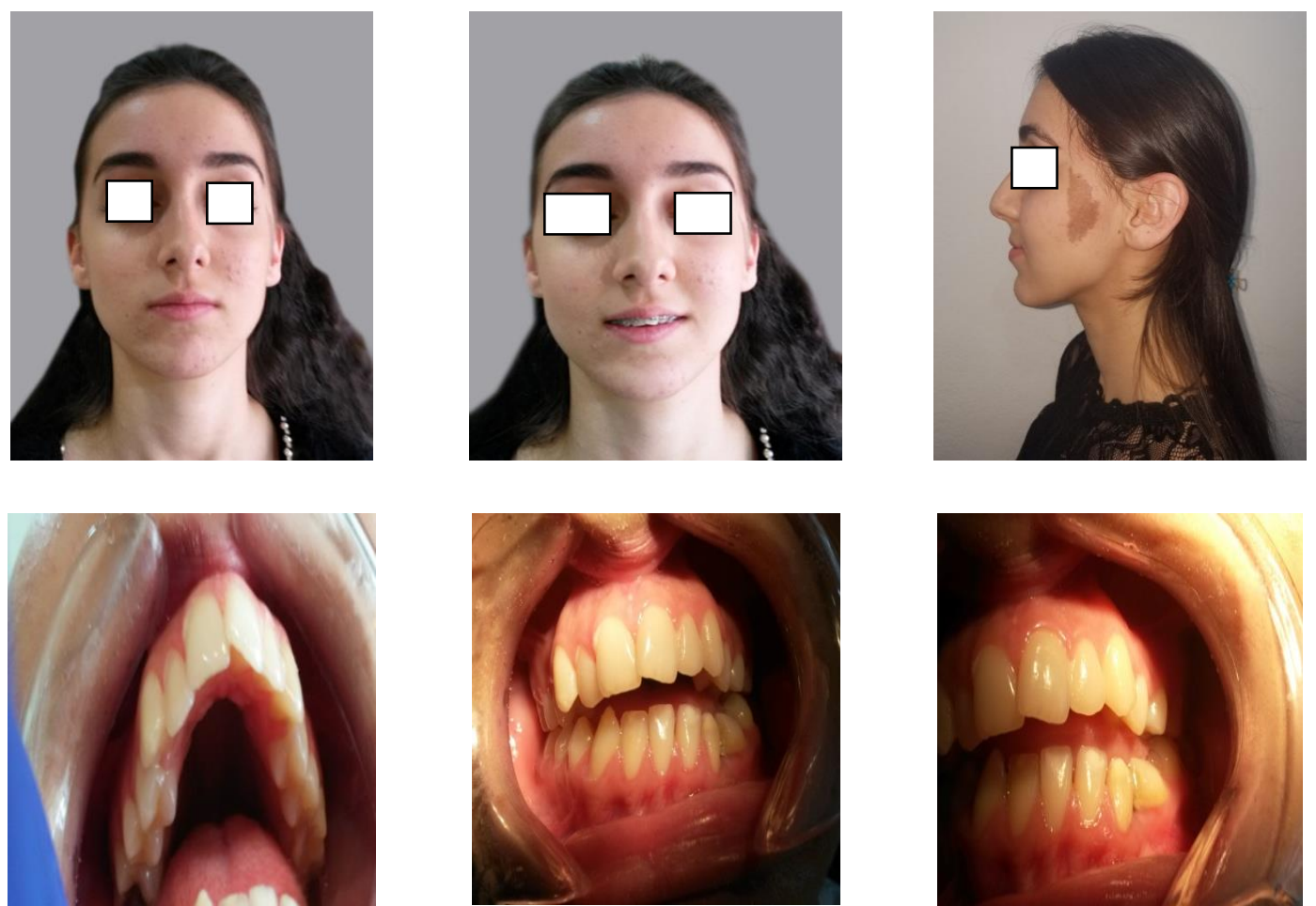

Figure (1): Pretreatment intraoral and extraoral photographs. 

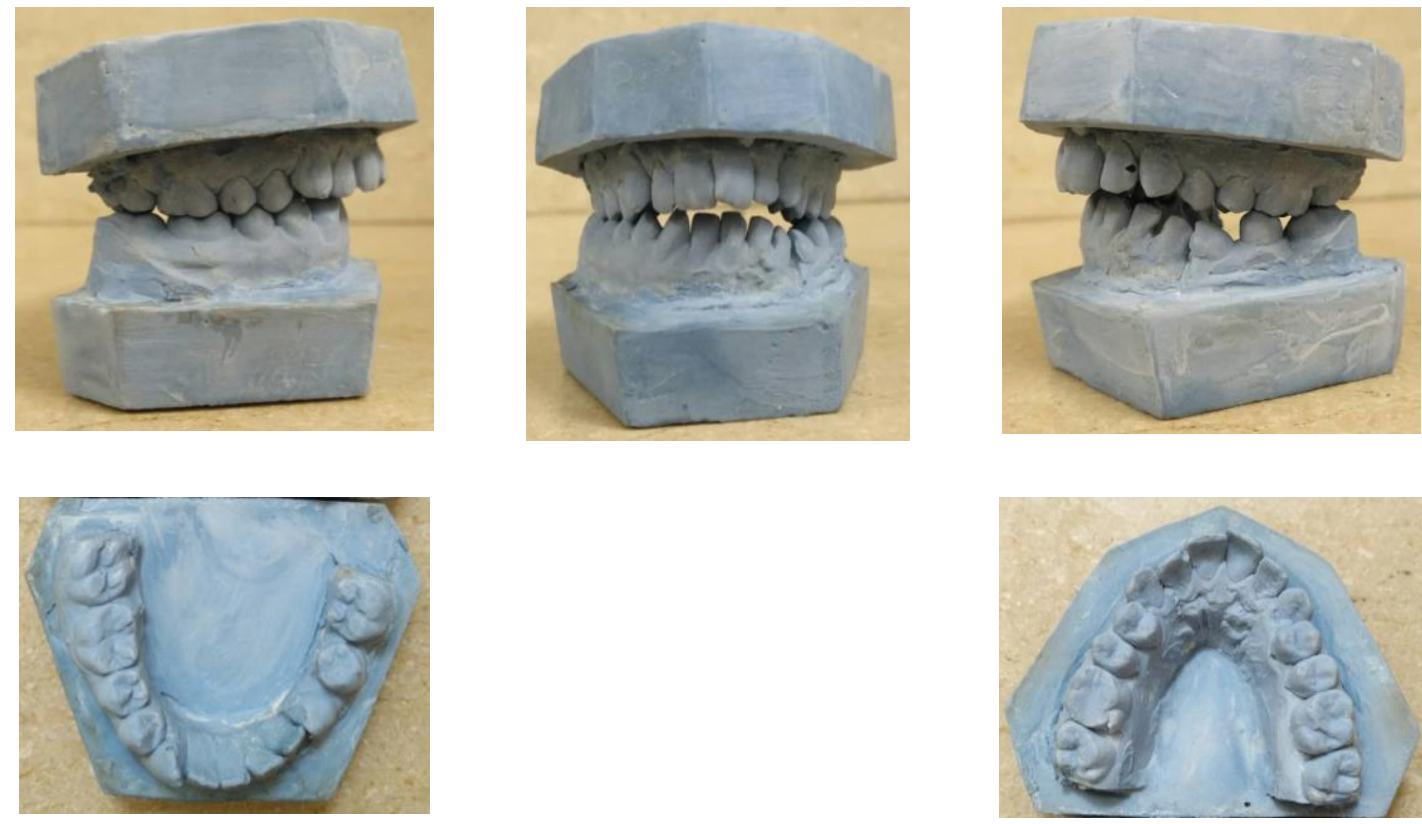

Figure (2): Pretreatment dental cast

Pre-treatment (T1) lateral cephalometric and panoramic radiograph(Figure 3) showed horizontally impacted mandibular left canine while periapical radiograph showed bifurcated roots with separated root canals as anatomical variation (Figure

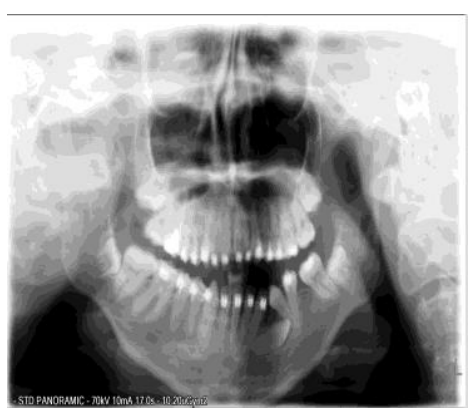

Figure (3): Pretreatment panoramic.and cephalometric radiograph
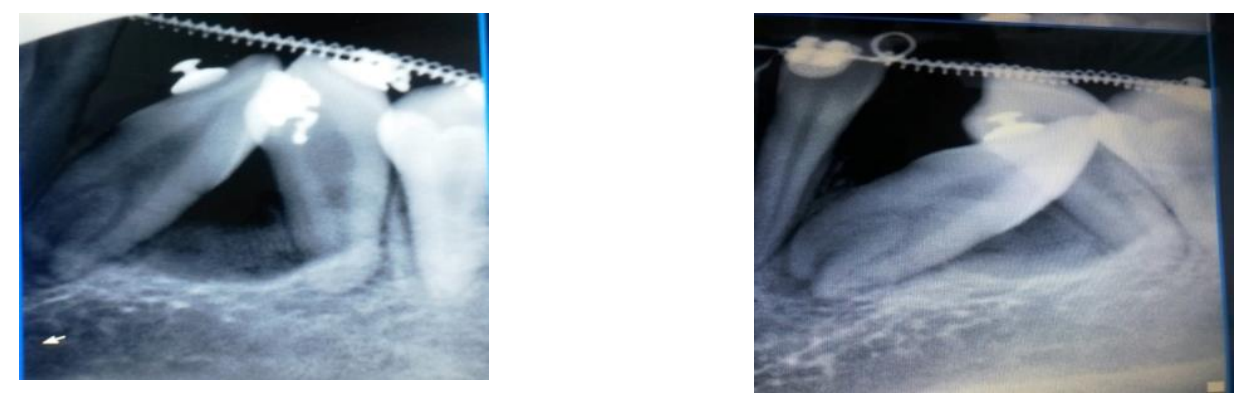

Figure (4): Periapical radiographs showed two roots with two canals as anatomical variation and the bottom bonded to impacted canine after surgical exposure. 
Table (1): Lateral cephalometric analysis for T1and T2

\begin{tabular}{lll}
\hline Measurements* $^{*}$ & T1 & T2 \\
\hline SNA angle & $78^{\circ}$ & $78^{\circ}$ \\
SNB angle & $75^{\circ}$ & $75^{\circ}$ \\
ANB angle & $3^{\circ}$ & $3^{\circ}$ \\
SN-maxillary plane angle & $7^{\circ}$ & $10^{\circ}$ \\
Upper incisor -maxillary plane angle & $115^{\circ}$ & $115^{\circ}$ \\
Lower incisor-mandibular plane angle & $90^{\circ}$ & $88^{\circ}$ \\
Inter-incisal angle & $123^{\circ}$ & $121^{\circ}$ \\
Maxillary-mandibular plane angle & $25^{\circ}$ & $25^{\circ}$
\end{tabular}

*All variables measured in degree for the Pre-treatment (T1)and immediate post-treatment (T2).

The treatment objectives in the sequence were as follows:

- Restore normal overbite and overjet.

- Alignment and leveling in the upper arch.

- Surgical exposure and bonding attachment on the impacted canine.

- Extrusion and uprighting the impacted and incomplete translocate mandibular left permanent canine.

- Preserving the periodontal health of the lower left permanent cane region following alignment.

- Achieve occlusal inter-cuspation with class I molar and canine relationship on both sides.

\section{Treatment Plan:}

In order to address the esthetic concern of the patient and upon her request, the decision was to bring the impacted canine into the normal position. The major risks and benefits including surgical procedure and possibility of gum recession were discussed with the patient and an informed consent form was taken before the start of the treatment.

\section{Progress of the treatment:}

Upper and lower fixed orthodontic appliance (Ortho Technology 0.022"X 0.028"Roth, stainless steel brackets) was bonded at the start of the treatment. The mandibular left first premolar was excluded during the alignment and leveling stage to prevent damage to its root from a possible collision with impacted canine.

The treatment started with aligning and leveling. The wire sequence included $0.14 "$ and $0.18 " \mathrm{NiTi}$, then this stage continued using 0.016" stainless steel (ST) arch wire till reach 0.019" X0.025" ST arch wire. An open coil spring was placed at this stage between the left lower lateral incisor and $2^{\text {nd }}$ premolar to regain the space for the impacted canine and the mesially 
inclined $1^{\text {st }}$ premolar (Figure 5,6). Following the regain of an adequate space, surgical exposure of impacted canine was commenced. The surgical procedure was carried out using a closed approach with a full thickness flap on the labial aspect of mandibular canine and premolar region under local anesthesia (Figure 5). Orthodontic button (Round base, Ortho Technology) was bonded on the lingual surface of the impacted canine and power chain elastic (short and clear, Ortho Technology) was attached to the button (Figure 7) and extended to a vertical coil with cantilever arm manually constructed and fitted on the lower arch wire distal to lateral incisor (Figure4,6). At this stage, orthodontic mini-screw $(6 \mathrm{~mm}$ Dentos, AbsoAnchor ${ }^{\circledR}$ Microimplants, selfdrilling) was placed between the upper left lateral incisor and canine to assist the anchorage demand in the lower arch and avoid intrusion of the lower incisors. A (3/16) intermaxillary elastic was connected to the mini-implant and extended to hook ligated on the bracket of the lower left lateral incisor (Figure 6). Within months, the cusp tip of the impacted canine emerged from the alveolar ridge. Extrusion of the canine continued until the full crown was exposed (Figure 7), at this stage, lower left first premolar orthodontic bracket was bonded and alignment of this tooth was achieved. The orthodontic button on the lingual surface of impacted canine was removed and the related orthodontic bracket was bonded on the labial surface of the canine to complete the alignment and leveling of lower dental arch. Following the full alignment and leveling of both arches, a box intermaxillary elastic( heavy 3/16 Ortho Technology) was applied to the anterior region to close the anterior open bite and achieve maximum intercuspation. Finally, the upper and lower dental arches were stabilized with $0.019 " \mathrm{X} 0.025^{\prime}$ ST arch wire (Figure 8).

Treatment duration was 32 months followed by upper and lower fixed appliances de-bonding. Coaxial ST wire 0.010 X 0.028" (Ortho Technology) bonded on the lingual surface of the lower incisors and canines as a fixed retainer (Figure 9). An upper removable appliance was constructed with two Adam clasps and Hawley labial arch to serve as an orthodontic retainer.

Figures $(9,10)$ showed the post treatment extra oral and intraoral radiographic views and dental cast model immediately following the removal of the fixed orthodontic appliances (T2). Figureure (11) demonstrates the post treatment panoramic and cephalometric radiograph views .Table (1) demonstrated the pre-treatment (T1) and post-treatment (T2) lateral cephalometric measurements.

Post treatment panoramic and periapical radiographic (Figure 11,12) finding shows normal position of the left mandibular canine. The radiographic view demonstrates normal alveolar contours and support. No obvious damage to the left first premolar and canine was seen although, there was mild gum recession and root 
shortening as a result of apical root resorption in the lower left canine.

Post treatment cephalometric measurements showed that the skeletal pattern remained normal and that the treatment approach and objectives were appropriate because all other cephalometric values were in the normal ranges. The only rele-
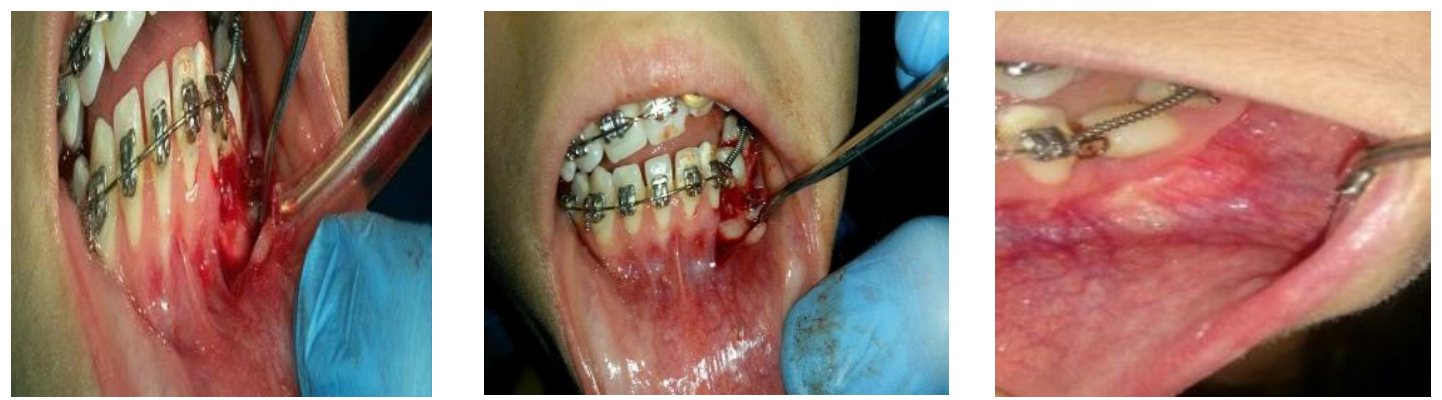

Figure (5): surgical exposure of impacted lower canine by close approaches

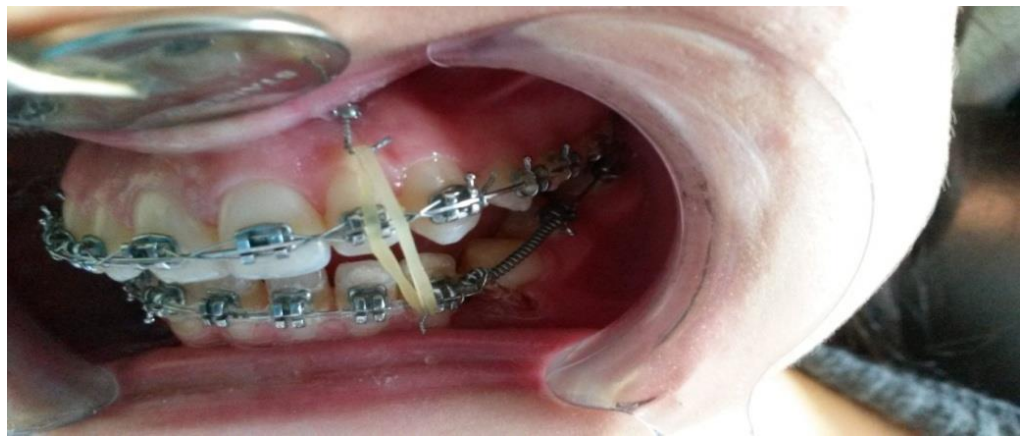

Figure (6): Mid treatment intraoral radiographs howed micrimplant with interarch elastic to increase anchorage.
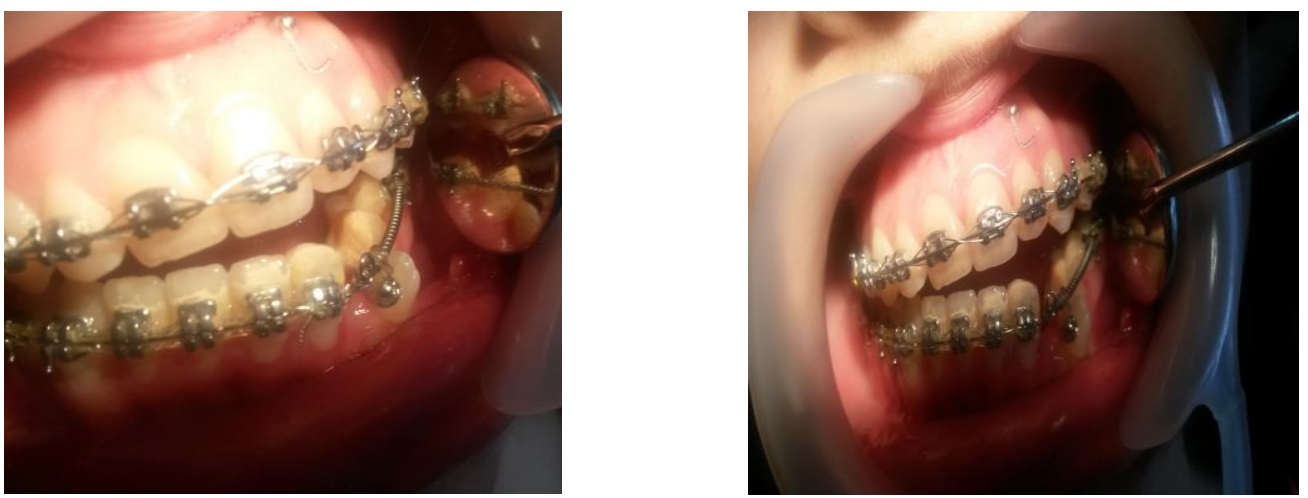

Figure (7): mid treatment intraoral radiograph showed the exposure of impacted canine inside the oral cavity 

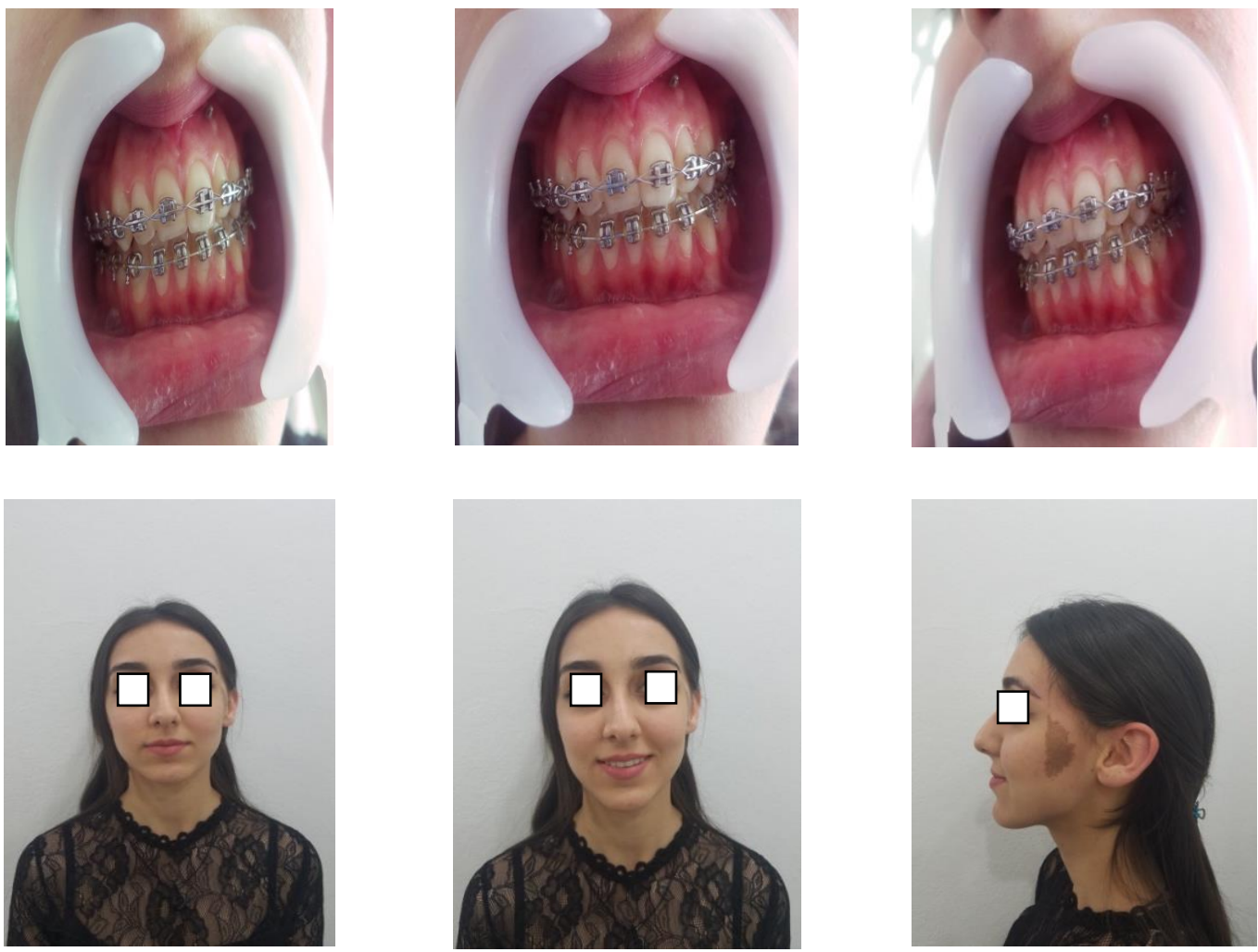

Figure (8) : intraoral radiograph showed the exposure upper and lower teeth stabilized with $0.019 "$ X $0.025 "$ ST arch wire.
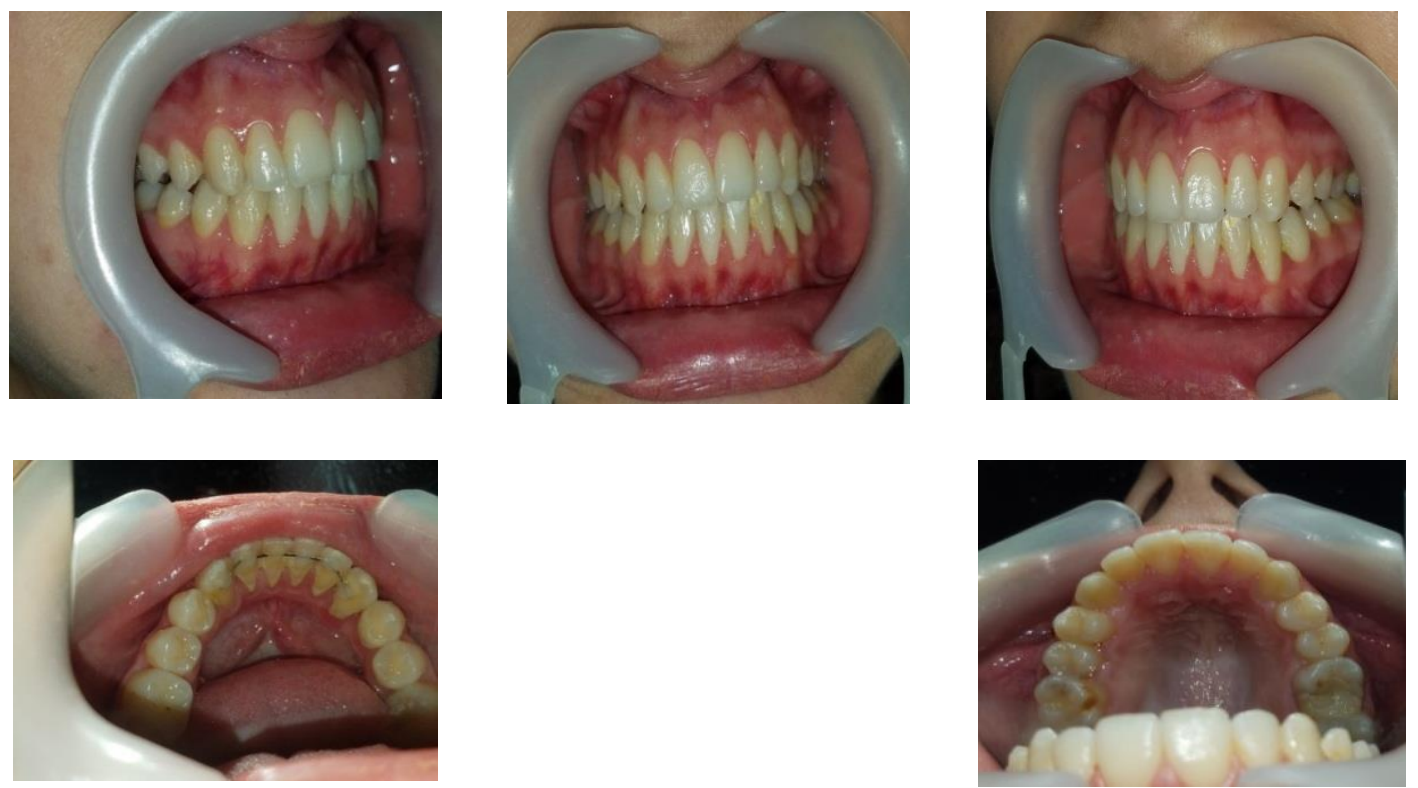

Figure (9): posttreatment intraoral and extraoral radiograph. 

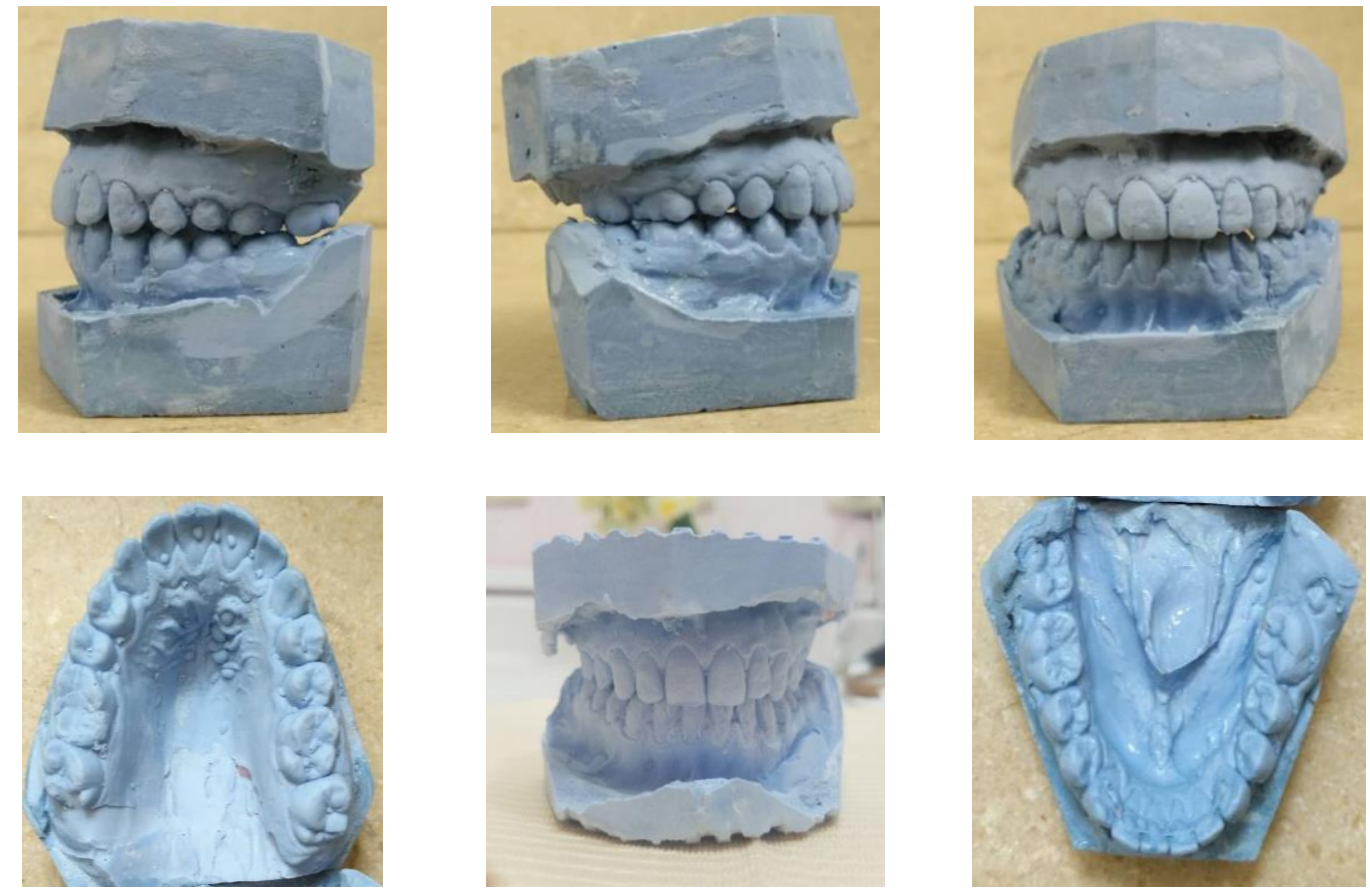

Figure (10): posttreatment dental cast
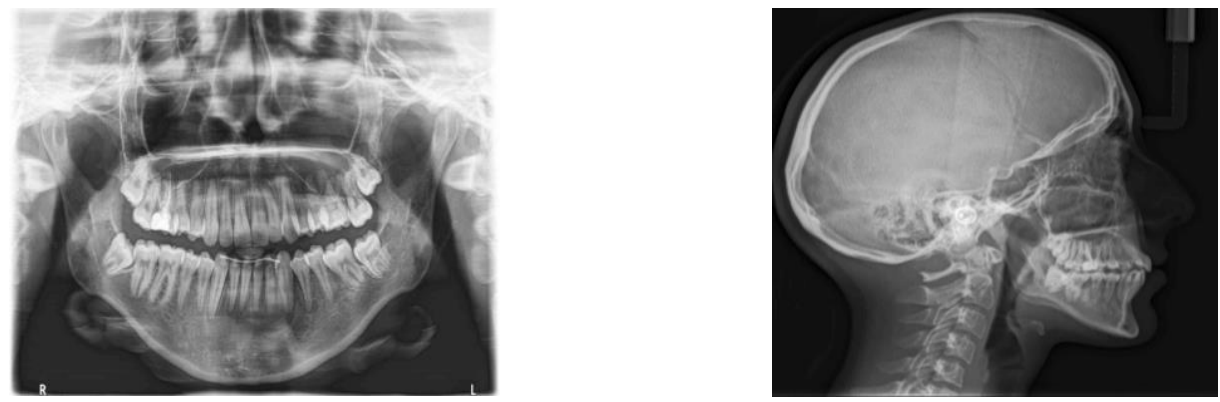

Figure (11): Post treatment panoramic. and cephalometric radiograph

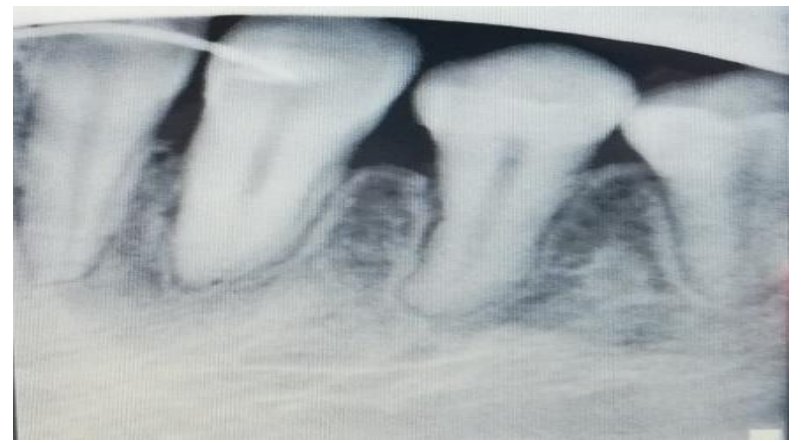

Figure (12): Post treatment periapical radiograph for the lower left canine.

\section{DISCUSSION}

This case report describes the orthodontic treatment and follow-up of an adult patient who was presented with horizontally impacted lower left canine with two roots (one facial and one lingual) as a 
dental anatomical anomaly in addition to anterior open bite malocclusion.

All teeth in the oral cavity could be impacted, however, it has been reported that third molars, maxillary canines, maxillary and mandibular premolars, and maxillary central incisors are the teeth most frequently involved ${ }^{(21) \text {. The }}$ prevalence of impacted maxillary canines is $0.9-2.2 \%$, but mandibular canine impaction occurs less frequently ${ }^{(22)}$.

Because of its location and anatomy, Maxillary and mandibular canines has been referred to as"Corner-stone" hence, every effort should be paid to avoid removal of impacted canine ${ }^{(23,24)}$.

Generally mandibular canine has one root canal, However, the presence of two roots and two separate canals has also been reported ${ }^{(20,25)}$. D'Amico et al (2003) concluded that the prevalence of these anatomic variations in mandibular canines is $1 \%$. This anatomical anomaly could be the main causative factor for impaction ${ }^{(26)}$.

The canine is situated in a strategic position between the anterior and posterior segment of the dental arch and plays an important role in functional occlusion and aesthetics ${ }^{(27)}$ also if not aligned can cause root resorption of adjacent teeth, a compromised occlusion and unpleasant appearance ${ }^{(28)}$.

Treatment of impacted and transposed mandibular canine is quite challenging. The mandibular alveolar bone is relatively narrow and available bucco-lingual dimension does not allow correction of transposition without interference with the roots of adjacent teeth. Owing to that fact and to avoid any damage to adjacent tooth root, the mandibular left first premolar was not included in the fix appliance treatment at the stages of alignment and leveling and the initial stage of canine uprighting. The mandibular left first premolar was included immediately after the crown of the impacted canine was retracted away from the roots of the premolar.

Microimplant (Temporary Anchorage Device TAD) was selected as a method of anchorage reinforcement. The TAD was inserted between the roots of upper left permanent canine and lateral incisor to counteract the intrusion force generated by the uprighting of lower canine. This was achieved by the application of intermaxillary elastics extended from the TAD to the hook on the lower left first premolar bracket.

The use of a closed approach flap for canine exposure enabled good oral hygiene to be maintained more readily and reduces post-operative discomfort. A mild gum recession and root resorption to the lower left canine were observed at the end of treatment. This was possibly due to bone inflammation associated with orthodontic tooth movement. In addition to that, minimal apical root resorption was detected, root resorption associated with orthodontic tooth movement has been frequently reported ${ }^{(29)}$.

Permanent wire retainer was bonded lingually from canine to canine in 
the lower arch, in addition to the removable orthodontic retainer in the upper arch to reduce relapse. 3 years follow up showed no relapse in both arches.

\section{CONCLUSIONS}

Despite the long treatment time and mild root resorption complexity of applied mechanics, the orthodontic extrusion and alignment of horizontally impacted incomplete transposition left mandibular canine showed successful results in both functional and esthetic improvement and should be considered for similar cases.

\section{REFERENCES}

1. Richardson $\mathrm{G} \&$ Russell KA. A review of impacted permanent maxillary cuspids diagnosis and prevention. J Canadian Dental Associ. 2000; 66(9):497501.

2. Peck S, Peck L \& Kataja M. The palatally displaced canine as a dental anomaly of genetic origin. The Angle orthodontist.1994; 64(4): 249-256.

3. Ma'Aita J \& Alwrikat A. Is the mandibular third molar a risk factor for mandibular angle fracture? Oral Surgery, Oral Medicine, Oral Pathology, Oral Radiology, and Endodontology. 2000;89(2):143-146.

4. D'Amico R M, Bjerklin K, Kurol J \& Falahat B. Long-term results of orthodontic treatment of impacted maxillary canines. The Angle orthodontist.2003;73(3): 231-238.

5. Aydin, U., Yilmaz, H., \& Yildirim, D. Incidence of canine impaction and transmigration in a patient population. Dentomaxillofacial Radiology.2004;33(3): 164-169.

6. Al-Ramil A M, Al-Wosaibi A M \& Bukhary M T. Prevalence of Impacted Teeth and Associated Pathologies : A Radiographic Study, Al Ahsa, Saudi Arabia Population. The Egyptian Journal of Hospital Medicine;2018;70 (12): 2130-2136.

7. Ashok D, Pradeep A, Ram Prasad Y, Arun Kumar M, Anjani Kumar Y, et al. Kissing canines associated with dentigerous cyst, a case report of transmigrated bilateral impacted mandibular canines. International Journal of Oral and Craniofacial Science.2017; 3(1): 014-016.

8. Plakwicz P, Czochrowska E \& Kukuła K . Impacted and transmigrant mandibular canines incidence, aetiology, and treatment: A systematic review. European Journal of Orthodontics. 2017; 40(2): 226-227.

9. Fonseca JR. Oral and maxillofacial surgery. Phailadelphia: W.B. Saunders.2002; (1):342-371.

10. Monika R, Mahesh G \& Nikhil M. Bilateral Mandibular Canine Impaction: A Rare Case Report. J Oral 
Health and Community Dent .2009; $3(2): 38-41$.

11. Milano M, Barrett L \& Marshall E. Extraction of a horizontally impacted mandibular canine through a genioplasty approach: Report of a case. J Oral and Maxillofacia Surgy.1996; 54(10):1240-1242.

12. Joshi MR. Transmigrant mandibular canines: a record of 28 cases and a retrospective review of the literature. The Angle orthodontist.2001;71(1):12-22.

13. Alberto PL.Management of the impacted canine and second molar, Oral Maxillofac. Surg. Clin. N. Am., 19 .2007; (19): 59-68

14. Hitchin AD.The impacted maxillary canine, Dent. Pract. Dent. Rec., 2 .1951; pp. 100-103.

15. GüdüzK, AçikgözA\& EğrioğluRad iologic E. Investigation of prevalence, associated pathologies and dental anomalies of non-third molar impacted teeth in Turkish oral patients, Chin. J. Dent. Res.2011; (14): 141-146

16. Bishara SE.Impacted maxillary canine: a review, Am. J. Orthod. Dentofac. Orthop .1992; (101): 159-171.

17. Bishara SE. Impacted maxillary canines: A review. Am J Orthod Dentofacial Orthop 1992;(101):159-71.
18. Versiani M A, Pécora J D \& Sousa-Neto M D. Microcomputed tomography analysis of the root canal morphology of single-rooted mandibular canines. Inter endodontic $J$. 2013;46(9): 800-807.

19. Roy D K, Cohen S, Singh V P, Marla V\& Ghimire S. Endodontic management of mandibular canine with two roots and two canals: A rare case report. BMC Research Notes, 2018; 11(1):111.

20. Victorino F R, Bernardes $\mathrm{R} A$, Baldi JV, Moraes IG, Bernardinelli N, Garcia R B\& Bramante CM. Bilateral mandibular canines with two roots and two separate canals: Case report. Brazilian Dental J. 2009; 20(1): 84-86.

21. Rajic S, Muretic Z \& Percac S. Impacted canine in a prehistoric skull. The Angle orthodontist. 1996;66(6): 477-480.

22. Jacoby H. The etiology of maxillary canine impactions. American J orthodontics.1983;84(2):125-132.

23. Sapna Y, Garg M, Shreya S \& Chauhan R.Balachandran, J., \& Kotwal, S. (2020). Endodontic management of mandibular canine having two roots: A rare presentation. $\mathrm{J}$ 
Dent \& Oral Disord.2020; 6(4): 1137.

24. Andrei OC, Mărgărit R \& Gheorghiu IM. Endodontic treatment of a mandibular canine with two roots. .Rom $J$ MorpholEmbryo. 2001;52(3):923-926.

25. D'arcangelo C, Varvara G \& Fazio P D. Root canal treatment in mandibular canines with two roots: A report of two cases. Inter Endodontic J.2001; 34(4): 331-334.

26. Bishara S E. Clinical management of impacted maxillary canines. Seminars in orthodontics.1998; 4(2): 87-98.
27. Zafarmand AH \& Gholam GA. Evalution of the periodontal status of palatally impacted maxillary canines after exposure using a modified window technique.World $J$ Orthod. Winter.2009;10(4):295-300.

28. Peerlings R H.Treatment of a horizontally impacted mandibular canine in a girl with a Class II Division 1 malocclusion. American J Orthodont Dentofacial Orthopedics.2010;137(4).

29. Apajalahti S \& Peltola J S. Apical root resorption after orthodontic treatment a retrospective study. The European J Orthodont.2007;29(4): 408-412. 